\title{
Anaesthetic management in Wilson's disease with severe neuropsychiatric manifestations
}

\author{
PR Nanjangud ${ }^{1^{*}}, S$ Prasad ${ }^{2}$ \\ Assistant Professor ${ }^{1}$, Junior Resident ${ }^{2}$, Kasturba Medical College, Manipal, India.
}

\begin{abstract}
A 15 year-old girl presented with acute psychosis, seizures and right shoulder dislocation with an overlying abscess. The symptomatology was diagnosed to be due to Wilson's disease. The anaesthetic management and postoperative analgesia was a challenging task in presence of hepatic and acute neurological dysfunction as well as difficulty in pain assessment. There are only a few reported cases of anaesthetic management in patients with Wilson's disease with severe neuropsychiatric symptoms. This case portrays the safe use of both general and regional anaesthesia in such a patient.
\end{abstract}

Keywords: Wilson's disease; neuro-psychiatric symptoms; anaesthesia; interscalene block

\section{Case Report}

A $15 \mathrm{yr}$ old, $30 \mathrm{~kg}$ girl presented with progressive slurring of speech, gait disturbance, insomnia and altered behaviour over a period of 20 days. She was admitted to a psychiatric hospital and started on risperidone and carbamazepine. A week later, she developed tightness of all four limbs and focal seizures. Quetiapine and thioridazine were added for better symptom control. She was then transferred to Kasturba Hospital, Manipal. There was no history of trauma, chronic fever or drug abuse. Perinatal period and development were within normal limits. There were no psychiatric/ genetic disorders or untimely deaths in her family. On admission, child had resting tremors, choreoathetoid movements, hypertonia of all four limbs and recurrent right sided focal seizures. Later, child was noted to have fluctuating consciousness, inattention and inappropriate laughter and crying. Examination revealed a swollen and dislocated right shoulder. A provisional diagnosis of

*Correspondence: PR Nanjangud

Email: pawannanjangud@gmail.com

0000-0001-6265-1878

Received: $10 / 04 / 2017$

Accepted: 08/05/2017

DOI: 10.4038 /slja.v25i2.8226



encephalopathy was made and further evaluation was performed. Biochemical tests revealed increased serum copper $(209 \mathrm{mcg} / \mathrm{dL})$ and urine copper levels $(373 \mathrm{mcg} / 24 \mathrm{~h})$ and mildly elevated liver enzymes in serum (Aspartate Transaminase75 IU/L, Alanine Transaminase-109 IU/L, Alkaline phosphatase-87 U/L). Computed tomographic scan of brain and cerebro-spinal fluid studies were normal. Toxicology and metabolic screening tests ruled out toxins and inborn errors of metabolism. Steroids and penicillamine were started with a presumptive diagnosis of Wilson's disease.

Trihexiphenidyl, quetiapine and levetiracetam were used to control her symptoms. A week after therapy, the swelling over the right shoulder was diagnosed to be a developing abscess. Orthopaedic surgeon scheduled her for closed/open reduction of the dislocation with drainage of abscess. Written informed consent was obtained from the parents for surgery and anaesthesia, after explaining the risk of perioperative adverse events.

Our initial plan was to provide an interscalene block. However, due to the fluctuating consciousness and recurrent choreo-athetoid movements, we proceeded with general anaesthesia. Electrocardiogram, non-invasive blood pressure and pulse oximetry was established and a $20 \mathrm{G}$ venous cannula secured and anaesthesia induced with fentanyl $60 \mathrm{mcg}$ and propofol $40 \mathrm{mg}$. Atracurium $15 \mathrm{mg}$ was used to facilitate tracheal intubation with $6.5 \mathrm{~mm}$ cuffed endotracheal tube. Anaesthesia was maintained with isoflurane $1 \%$ 
with $66 \%$ nitrous oxide in oxygen and analgesia was with fentanyl boluses and intravenous paracetamol 450mg. Abscess drainage and open reduction lasted for 90 minutes and the patient remained haemodynamically stable throughout. For postoperative analgesia, an ultrasound guided interscalene brachial plexus block was given at the end of surgery with $15 \mathrm{ml}$ of $0.25 \%$ bupivacaine using a $20 \mathrm{G}$ nerve stimulator needle. Inhalational agents were discontinued, neuromuscular blockade reversed with neostigmine $1.5 \mathrm{mg}$ and glycopyrrolate $0.3 \mathrm{mg}$ and trachea extubated following spontaneous eye opening, movement of all limbs, return of airway reflexes and intermittent response to commands. She was shifted to the paediatric intensive care unit for monitoring. No deterioration of neurological symptoms or liver function was noted during the next $48 \mathrm{~h}$. Delirium was assessed using CAM-ICU chart. On further follow up, she was fully conscious and cooperative with decreased extrapyramidal symptoms and improved speech while on penicillamine and levetiracetam.

\section{Discussion}

The presenting symptoms of delirium and involuntary movements in this case led to three differential diagnoses viz. Wilson's disease, septic encephalopathy and adverse drug reaction (to antipsychotic and antiepileptic drugs).

The most common causes of encephalopathy like metabolic disturbances and electrolyte imbalances were ruled out. Her medications were changed to rule out adverse reactions. She was found to satisfy most of the diagnostic criteria for Wilson's disease except for presence of Kayser-Fleischer (KF) ring, found in $95 \%$ of patients with neurological features of Wilson's disease. Though the rise in urine copper after penicillamine challenge was less than five times $(373 \mathrm{mcg} / 24 \mathrm{~h}$ to $1200 \mathrm{mcg} / 24 \mathrm{~h})$ in this patient, we did not perform a genetic test or liver biopsy as they do not confirm the diagnosis with $100 \%$ certainity. ${ }^{1}$ Since most features were suggestive of Wilson's disease, she was managed accordingly. The shoulder abscess was probably a contributing factor to the neurological symptoms. Hence, it required urgent drainage even in the presence of neuropsychiatric symptoms. ${ }^{2}$
Wilson's disease rarely presents with acute psychosis. ${ }^{3}$ Here the child presented with acute psychosis and was posted for a semi-emergent surgery before resolution of symptoms. Elective surgery is deferred in such cases till the resolution of symptoms to avoid worsening. ${ }^{4}$ However there is no literature to support the same when the aetiology of psychosis is Wilson's disease. To our knowledge, this is the first reporting of anaesthetic management of such a case.

Wilson's disease presents with a spectrum of symptoms ranging from neurological, hepatic, psychiatric or osteomuscular. ${ }^{5}$ Providing anaesthesia to such patients is challenging owing to detrimental effects of anaesthetic agents on liver function and neurological status. Our chief concerns were altered pharmacokinetics and possible deterioration of pre-existing neuropsychiatric symptoms.

Our plan to perform the procedure under interscalene block was to minimize haemodynamic alterations, avoid drugs requiring hepatic metabolism and to minimize the risk of neurologic deterioration. This was supported by a report by De Souza $\mathrm{H}$, which describes the use of interscalene block for rotator cuff repair without any complications. ${ }^{6}$ Administration of block was difficult due to delirium and choreo-athetoid movements that could not be controlled with sedation. Hence the procedure was performed under general anaesthesia.

There are few reported cases of general anaesthesia in patients with Wilson's disease without causing any hepatic or renal dysfuntion. ${ }^{7,8}$ Drugs with extra hepatic metabolism such as remifentanil, propofol, cis-atracurium and atracurium are safe in presence of hepatic dysfunction. ${ }^{9}$

Postoperative pain management was challenging in view of altered drug metabolism. Opioids were restricted due of possibility of accumulation and resultant respiratory depression. Also, they interfere with neurological assessment and can aggravate existing symptoms. ${ }^{9}$ We avoided NSAIDs due to their propensity to cause renal dysfunction. Hence, an interscalene brachial plexus block was given for postoperative analgesia to reduce systemic analgesics. The key point against 
block was the risk of nerve damage as it was performed under anaesthesia and due to the rare possibility of polyneuropathy in Wilson's disease that may or may not be related to penicillamine treatment. ${ }^{9} \quad$ Ultrasound guidance enabled visualization of neural structures and adequate spread of local anaesthetic around the plexus.

Another concern was pain assessment in postoperative period. There are several pain rating scales defined in paediatric age group and in patients with cognitive impairment like CHEOPS, FLACC, Baker-Wong FACES and COPT. ${ }^{9}$ The use of these scales become difficult in patients with severe psychotic symptoms, limb rigidity and labile affect. Hence, the adequacy of block was primarily judged by vital parameters and observation of behavioural changes ${ }^{10}$. Regional anaesthesia helps in decreasing systemic analgesic requirement and prevents worsening of delirium in patients with severe neuropsychiatric features of Wilson's disease.

\section{References}

1. Ferenci P, Caca K, Loudianos G et al. Diagnosis and phenotypic classification of Wilson disease. Liver International.2003;23:139-142. https://doi.org/10.1034/j.1600-0676.2003.00824.x PMid:12955875

2. Chaudhry N, Duggal AK. Sepsis Associated Encephalopathy. Advances in Medicine. 2014, Article ID 762320, 16 pages, 2014

3. Grover S, Sarkar S, Jhanda S, Chawla Y. Psychosis in an adolescent with Wilson's disease: A case report and review of the literature. Indian J Psychiatry. 2014;56:395-398.

PMid:25568485 PMCid:PMC4279302

4. Molnar G, Fava GA. Principles of medical psychiatry. Orland: Grune and Stratton Inc; 1987. Intercurrentmedical illness in the schizophrenic patients; p 451.

5. Ferenci P, Czlonkowska A, Stremmel W et al. EASL Clinical Practice Guidelines: Wilson's disease. J Hepatology. 2012;56:671-685.

6. De Souza Hobaika AB. Anaesthesia for a patient with Wilson's disease. Middle East J Anesthesiol. 2008;19(4):905-908.

PMid:18630776

7. Baykal M, Karpolat S. Anesthetic management of a Pediatric Patient with Wilson's disease. J Clin Med Res.April2010;2(2):99-101. https://doi.org/10.4021/jocmr2010.04.292w

8. Jung K, Ahn T, Jeon BS. Wilson Disease with an Initial Manifestation of Polyneuropathy. Arch Neurol.2005;62(10):1628-1631. https://doi.org/10.1001/archneur.62.10.1628 PMid:16216950

9. Rahimzadeh P, Safari S, Faiz SHR, Alavian SM. Anesthesia for Patients with Liver Disease. Hepatitis Monthly.2014;14(7):e19881. https://doi.org/10.5812/hepatmon.19881

10. Stites M. Observational Pain Scales in Critically Ill Adults. Crit Care Nurse.2013;33(3):68-78. https://doi.org/10.4037/ccn2013804

PMid:23727853 